\title{
Detection of nerve fibers in the eutopic endometrium of women with endometriosis, uterine fibroids and adenomyosis
}

\author{
Garima Yadav, MS¹, Meenakshi Rao, MD², Meenakshi Gothwal, MS ${ }^{1}$, Pratibha Singh, MD¹, \\ Priyanka Kathuria, $\mathrm{MS}^{1}$, Prem Prakash Sharma, $\mathrm{MD}^{3}$ \\ ${ }^{1}$ Department of Obstetrics \& Gynaecology, ${ }^{2}$ Department of Pathology, ${ }^{3}$ Department of Community Medicine and Family Medicine, All India Institute of \\ Medical Sciences, Jodhpur, India
}

\section{Objective}

The primary objective of this study was to establish the presence of nerve fibers in the eutopic endometrium of women with endometriosis and to determine whether these nerve fibers are exclusive to endometriosis or are also found in other pelvic pathologies associated with dysmenorrhea.

\section{Methods}

Endometrial tissue was obtained by aspiration (Pipelle), endometrial curettage, or following hysterectomy in women with endometriosis confirmed through histopathological examination, leiomyomas, and adenomyosis. The eutopic endometrium was subjected to immunohistochemical staining to detect PGP 9.5, which is a highly specific pan-neuronal marker. The nerve fiber density was correlated with the patient's pain score, as indicated by the Visual Analog Scale. A control group was formed by staining the endometrium of women presenting with dysmenorrhea but without the above-mentioned disorders.

\section{Results}

Nerve fibers were observed in sections of the endo-myometrium (in the deep endometrium) in $68 \%$ of patients with endometriosis who underwent hysterectomy or a deep endometrial biopsy. Nerve fibers were not observed in the aspirated endometrium of women with endometriosis. Only $13.7 \%$ of women with adenomyosis and $3.3 \%$ of women with fibroids had nerve fibers in their endometrium. Nerve fiber density was correlated with pain score in women with endometriosis.

\section{Conclusion}

Nerve fibers were found in the functional layer of eutopic endometrium in women with endometriosis; hence, we concluded that the presence of nerve fibers in the eutopic endometrium could diagnose endometriosis with a fairly good specificity of $92.7 \%$. However, the absence of nerve fibers does not always exclude the disease.

Keywords: Adenomyosis; Dysmenorrhea; Endometriosis; Leiomyoma; Nerve fibers

\section{Introduction}

Endometriosis is a common gynecological disorder with a high degree of variability in the age of onset, severity and extent of pain symptoms, anatomical locations, and response to treatment. The prevalence of endometriosis in teenage girls with pelvic pain is as high as $50-60 \%$, while in infertile women, it can be up to $50 \%[1,2]$. Moreover, the symptoms are not specific for endometriosis and can be seen in other
Received: 2021.04.01. Revised: 2021.05.06. Accepted: 2021.06.09. Corresponding author: Garima Yadav, MS

Department of Obstetrics \& Gynaecology, All India Institute of Medical Sciences, Basni Industrial Area, MIA 2nd Phase, Basni, Jodhpur 342001, India

E-mail: garimapunein@gmail.com https://orcid.org/0000-0002-1268-6973

Articles published in Obstet Gynecol Sci are open-access, distributed under the terms of the Creative Commons Attribution Non-Commercial License (http://creativecommons. org/licenses/by-nc/3.0/) which permits unrestricted non-commercial use, distribution, and reproduction in any medium, provided the original work is properly cited.

Copyright $\odot 2021$ Korean Society of Obstetrics and Gynecology 


\section{Obstetrics \& Gynecology Science}

Garima Yadav, et al. Nerve fibers in eutopic endometrium

diseases as well. In many women with endometriosis, no abnormality is detected during clinical examination [3], while uterosacral tenderness or palpable ovarian cysts may suggest endometriosis. Among the imaging studies for endometriosis, a recent Cochrane review concluded that transvaginal ultrasound, computed tomography, and magnetic resonance imaging (MRI) have limited diagnostic importance in the early stages of the disease, and these modalities cannot diagnose peritoneal endometriosis [4]. The routine use of MRI is also limited by its cost. The gold standard tool for diagnosing endometriosis is histological confirmation using laparoscopy. In many situations, performing an invasive test such as laparoscopy for diagnosing a condition is not cost-effective and mandates a patient to wait for a considerable time before endometriosis is confirmed.

The mechanism of pain generation is not well understood, although it is recognized that endometriosis is an inflammatory condition with multiple leucocytes of different types in endometriotic plaques and eutopic endometrium [5]. Hence, there is a potential for the local production of pain-stimulating molecules within this tissue from leucocytes, but limited information is available on pain stimulation pathways for the mechanism of sensory nerve activation in eutopic endometrium or endometriotic plaques.

Recent studies have observed that fine, unmyelinated sensory nerve fibers are present in the functional layer of the eutopic endometrium in women with endometriosis. These nerve fibers have also been observed in the ectopic plaques of peritoneal endometriosis. Further studies have refuted the above results and shown diverse findings, such as the presence of nerve fibers in women without endometriosis or complete absence of nerve fibers in women with endometriosis [6-8]. These nerve fibers are detected by immunohistochemical methods using various neuronal markers as antigens; the most specific of them being protein gene product 9.5 (PGP 9.5), which detects sensory nociceptive neurons. The demonstration of PGP 9.5 antigen-positive neurons is performed using a rabbit-monoclonal PGP 9.5, and the presence of these nerve fibers in the endometrium is postulated to be responsible for endometriosis-associated pain. Thus, this study attempted to provide a simple and reliable test to diagnose endometriosis and at the same time explain the pathogenesis of pain generation in endometriosis. We sought to detect PGP 9.5 antigen-positive nerve fibers in patients with chronic pelvic pain or dysmenorrhea with a confirmed diagnosis of uterine fibroids or adenomyosis.

\section{Materials and methods}

This prospective study was conducted over a period of 1 year. A total of 247 women of reproductive age who presented with dysmenorrhea or chronic pelvic pain, diagnosed with endometriosis/fibroid/adenomyosis or no disease on imaging and with surgical treatment scheduled were approached for the study. Among them, 190 patients with histopathological confirmation of endometriosis, adenomyosis, or uterine fibroids were included in the study. There were 30 other women included in the study, who underwent hysterectomy for abnormal uterine bleeding or uterine prolapse and had variable degrees of dysmenorrhea but no evidence of any abovementioned pathologies. This study included 220 patients. Among the 190 patients, 56 (29.5\%) had endometriosis, 73 (38.4\%) had adenomyosis, and 61 (32.1\%) had uterine fibroids. Among the 56 women with endometriosis, 5 women had focal adenomyosis and 14 women who underwent surgery for adenomyosis had co-existent intramural small fibroids. All women were in the reproductive age group, with one of the presenting symptoms of dysmenorrhea and/or chronic pelvic pain.

Women with suspected endometriosis on preoperative imaging studies were scheduled for diagnostic and operative laparoscopy. Surgical staging of the disease was performed according to the Revised American Society of Reproductive Medicine classification [9], and the pain score of the patient was calculated using the 10-point Visual Analog Scale (VAS), wherein a score of 0 was assigned to no pain and a score of 10 was assigned to agonizing pain. The endometrial tissue was sampled using Novak's curette in all women with a visual evidence of endometriosis on laparoscopy, and a single full-thickness strip of the endometrium was retrieved. The endometrium of only women with endometriosis confirmed through histopathological examination was subjected to further immunohistochemistry (IHC) staining. A few cases of endometriosis underwent endometrial aspiration using a Pipelle endometrial sampler (10 women) in the outpatient setting, as these were referred to our hospital after being operated for endometriosis outside, but were still symptomatic with the disease and had recurrent endometriosis on imaging, although the pathological diagnosis was reviewed at our 


\section{Obstetrics \& Gynecology Science}

Vol. 64, No. 5, 2021

center. Another subset of women underwent hysterectomy for stage III/IV endometriosis, and their endometrium was directly recruited from the hysterectomy specimens; hence, endometrial samples from both the proliferative and secretory phases of the cycle were available for IHC. In all cases of adenomyosis and fibroids, the endometrium was recruited only from the hysterectomy specimen to be sure of the pathology. The endometrium from the hysterectomy specimens without any evidence of endometriosis, adenomyosis, or fibroids was evaluated for the presence of nerve fibers, provided that their clinical records showed some degree of dysmenorrhea as one of the chief complaints, and endometriosis was ruled out from the patient's preoperative work-up and intraoperative notes.

Women who had undergone hormonal therapy for symptomatic treatment of endometriosis in the last 3 months were excluded from the study as hormonal therapy is said to decrease the number of nerve fibers in the functional layer of the endometrium. Women already operated for endometriosis and with no evidence of recurrent disease on imaging were also excluded, as surgical excision of endometriosis is expected to decrease the expression of nerve growth factors in the eutopic endometrium. Women with acute pelvic inflammatory disease, suspected pregnancy, genital malig- nancies, undiagnosed vaginal bleeding, documented genital tuberculosis, and post-menopausal women undergoing hysterectomy for any benign condition were excluded from the study.

IHC was performed in an automated Leica Bondmax IHC stainer to detect nerve fibers in the endometrium using antibody PGP 9.5 (Polyclonal rabbit anti-PGP 9.5, Dako, Jena, Germany), at a dilution of 1:750. The fibroadipose tissue

Table 1. The mean age and VAS score of all the study participants

\begin{tabular}{lccc}
\hline $\begin{array}{l}\text { Type of } \\
\text { pathology }\end{array}$ & $\begin{array}{c}\text { Number of } \\
\text { patients }\end{array}$ & Age (yrs) & VAS \\
\hline Endometriosis & 56 & $34.8 \pm 4.56$ & $5.80 \pm 2.538$ \\
Adenomyosis & 73 & $36.2 \pm 6.25$ & $4.161 \pm 3.023$ \\
Uterine fibroids & 61 & $39.2 \pm 3.68$ & $3.91 \pm 3.214$ \\
$\begin{array}{l}\text { Control group } \\
\text { (dysmenorrhoea }\end{array}$ & 30 & $42.6 \pm 2.86$ & $3.56 \pm 1.26$ \\
$\quad$ but no pathology) & & & \\
Total ${ }^{\text {a) }}$ & 220 & & \\
\hline
\end{tabular}

Values are presented as mean \pm standard deviation unless otherwise indicated.

VAS, Visual Analog Scale.

${ }^{a)} F$-value: $5.412, P$-value: $<0.001$; as per one-way analysis of variance.

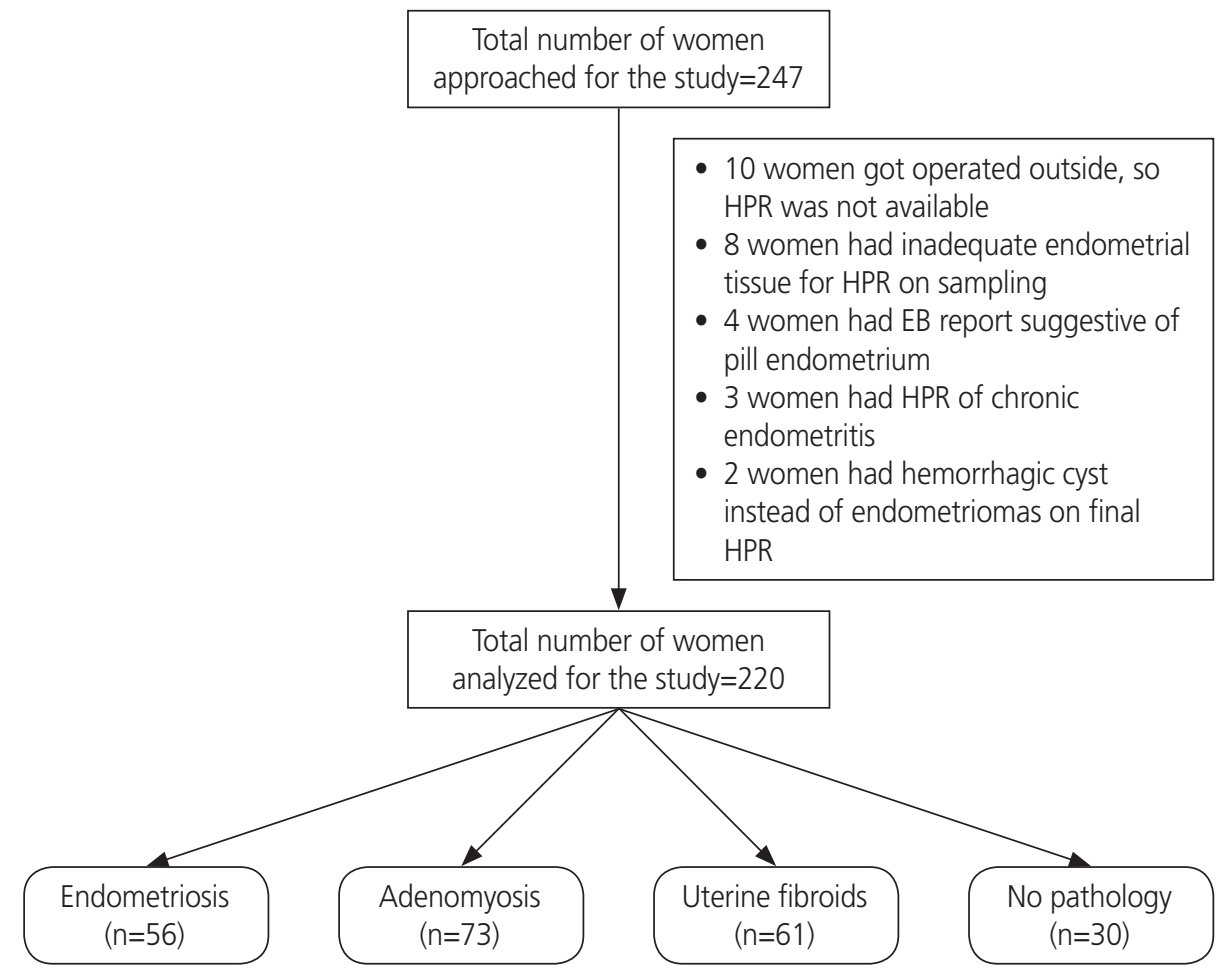

Fig. 1. Patient flow following recruitment in the study. HPR, histopathological report; $E B$, endometrial biopsy. 


\section{Obstetrics \& Gynecology Science}

Garima Yadav, et al. Nerve fibers in eutopic endometrium

containing microscopically visible nerve fibers on hematoxylin and eosin staining was used as a positive control. IHC was performed in sections of the endomyometrium in all cases of leiomyomata and adenomyosis diagnosed through histological examination. In cases of endometriosis, IHC was performed on sections of endo-myometrium in cases where hysterectomy had been performed, and in endometrial aspirates or endometrial biopsy tissues where hysterectomy had not been performed. All slides were observed under a microscope, Eclipse Ci-L (Nikon, Tokyo, Japan), for the evaluation of nerve fibers in the endometrium, and the density of nerve fibers was calculated by an expert pathologist (M.R) who was blinded to the initial diagnosis of endometriosis. The presence of nerves was defined whenever the whole morphology with multiple fascicles and epineurium was identified or when a single fascicle with the epineurium was seen along with positive $\mathrm{IHC}$ results. The nerve fiber density was calculated as the number of nerve fascicles/high-power field (HPF) ( $\times 400$ magnification, in a microscope field diameter of

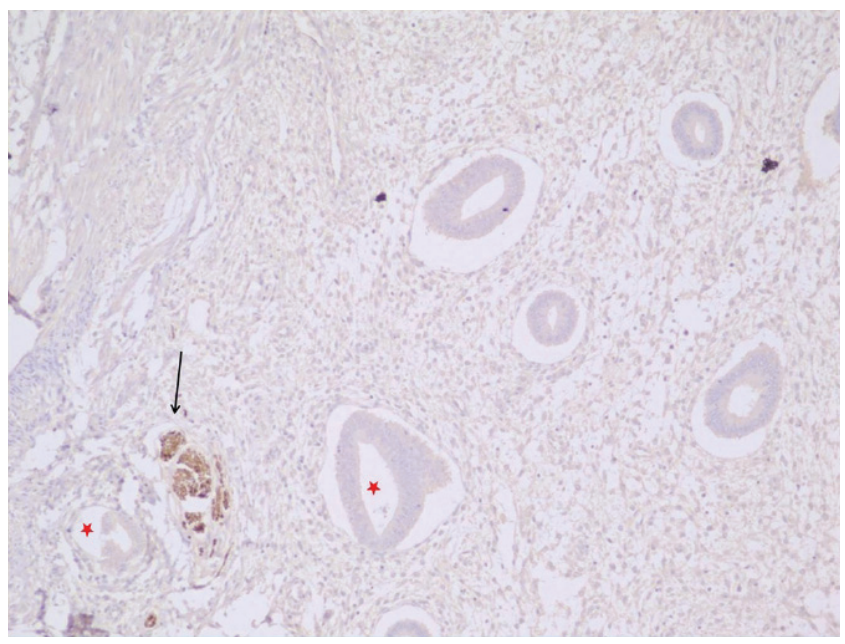

Fig. 2. The endometrium shows nerve fibers highlighted by the protein gene product (PGP) 9.5 antibody (black arrow) and endometrial gland (red asterisks) in a case of endometriosis (immunohistochemical staining, $\times 40$ ).
$0.5 \mathrm{~mm}$ ). The number of nerve fascicles was counted in $10 \mathrm{HPF}$, and the average number of nerve fibers per HPF was calculated.

\section{Statistical analysis}

Data were analyzed using SPSS software (version 21; IBM Corp., Armonk, NY, USA). Comparison of frequencies in the groups were compared using the chi-squared test, and $P<0.05$ was considered significant. The Spearman rank correlation coefficient was calculated between the VAS score for pain and the density of nerve fibers in the endometrium.

\section{Results}

The patient flow from recruitment to analysis is shown in Fig. 1. The mean age and VAS scores of all patients are presented in Table 1. Women with endometriosis had the highest mean VAS score, followed by women with adenomyosis, with a

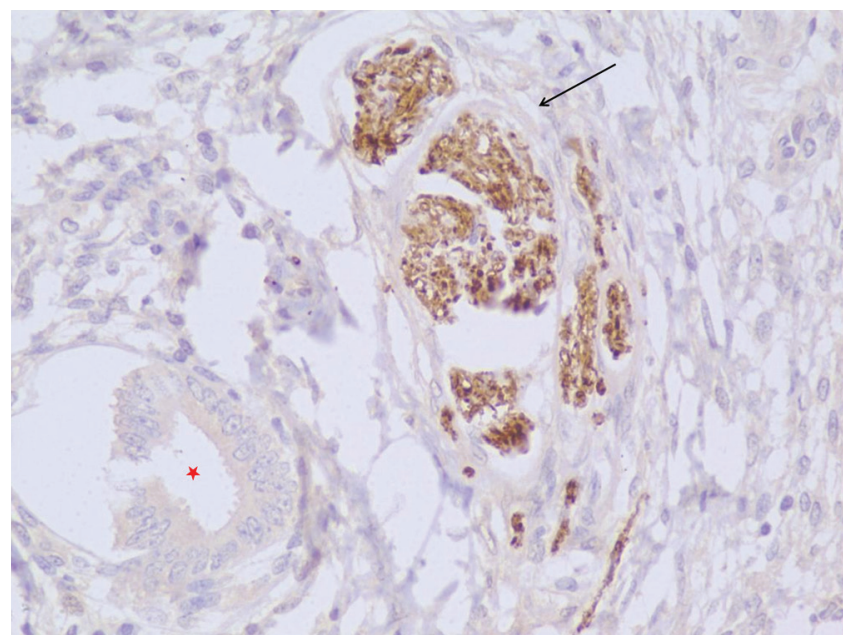

Fig. 3. High power view (immunohistochemical staining, $\times 40$ ) of a proliferative endometrium (red asterisk) showing nerve fibers highlighted by the protein gene product (PGP) 9.5 antibody (black arrow) in a case of endometriosis.

Table 2. The percentage of women with endometriosis with positive nerve fibers in the hysterectomy, curettage, and aspiration groups

\begin{tabular}{lcc}
\hline Method of collection & Number of positive nerve fibers cases & Number of cases without nerve fibers \\
\hline Hysterectomy & $20(64.51)$ & $11(35.49)$ \\
Endometrial currettage & $11(73.33)$ & $4(26.66)$ \\
Endometrial aspiration using Pipelle & 0 & $10(100)$
\end{tabular}

Values are presented as number (\%). 


\title{
Obstetrics \& Gynecology Science
}

\author{
Vol. 64, No. 5, 2021
}

$P$-value of $<0.001$ and $F$ ratio of 5.412 , as calculated by oneway analysis of variance.

\section{Distribution of stages of endometriosis}

Among the 56 cases of endometriosis confirmed through histological examination, 18/56 (32.14\%) women had stage I disease, 10/56 (17.85\%) had stage II disease, 19/56 (33.92\%) had stage III endometriosis, and the remaining 9/56 women (16.07\%) had stage IV disease. Five women had associated evidence of focal adenomyosis, but since the predominant pathology was endometriosis, these were considered in the endometriosis group. No correlation was found between the stage of endometriosis and the presence of nerve fibers.

\section{Procedures used to collect the endometrial tissue}

Among 56 cases of endometriosis, 31 patients underwent hysterectomy and bilateral salpingo-oophorectomy; therefore, IHC was performed on sections of the intact endomyometrium. The remaining 25 patients underwent only endometrioma excision along with excision or ablation of the peritoneal deposits with or without adhesiolysis. Among these 25 cases, IHC was performed on the endometrial aspirates in 10 cases and curettage tissue in 15 cases. In the remaining 134 women with adenomyosis or fibroid uterus,
IHC was performed on the full-thickness endometrium, including the endo-myometrial junction as they all underwent hysterectomy.

\section{Nerve fibers in the endometrium}

A total of $31 / 56$ (55.4\%) of women in the endometriosis group had nerve fibers compared to $10 / 73(13.7 \%)$ in the adenomyosis group, and 2/61 (3.3\%) in the fibroids group and none in the other groups (dysmenorrhea without any documented pathology on imaging or histopathological examination) had nerve fibers. There was a significant difference in the presence of nerve fibers in these groups $\left(\chi^{2}=50.6\right.$, $P<0.001)$, with a high number of women with endometriosis having endometrial nerve fibers.

\section{IHC findings}

In women with endometriosis, the nerve fibers highlighted by the PGP 9.5 antibody were observed only in the deeper layers of the functional endometrium (Figs. 2 and 3). Nerve fibers were seen in $20 / 31$ cases $(64.51 \%)$ of endometriosis who underwent hysterectomy. 11/15 (73.33\%) cases of endometriosis in whom endometrial curettage was performed nerve fibers were noted whereas none of the 10 cases in whom endometrial aspirate was taken showed
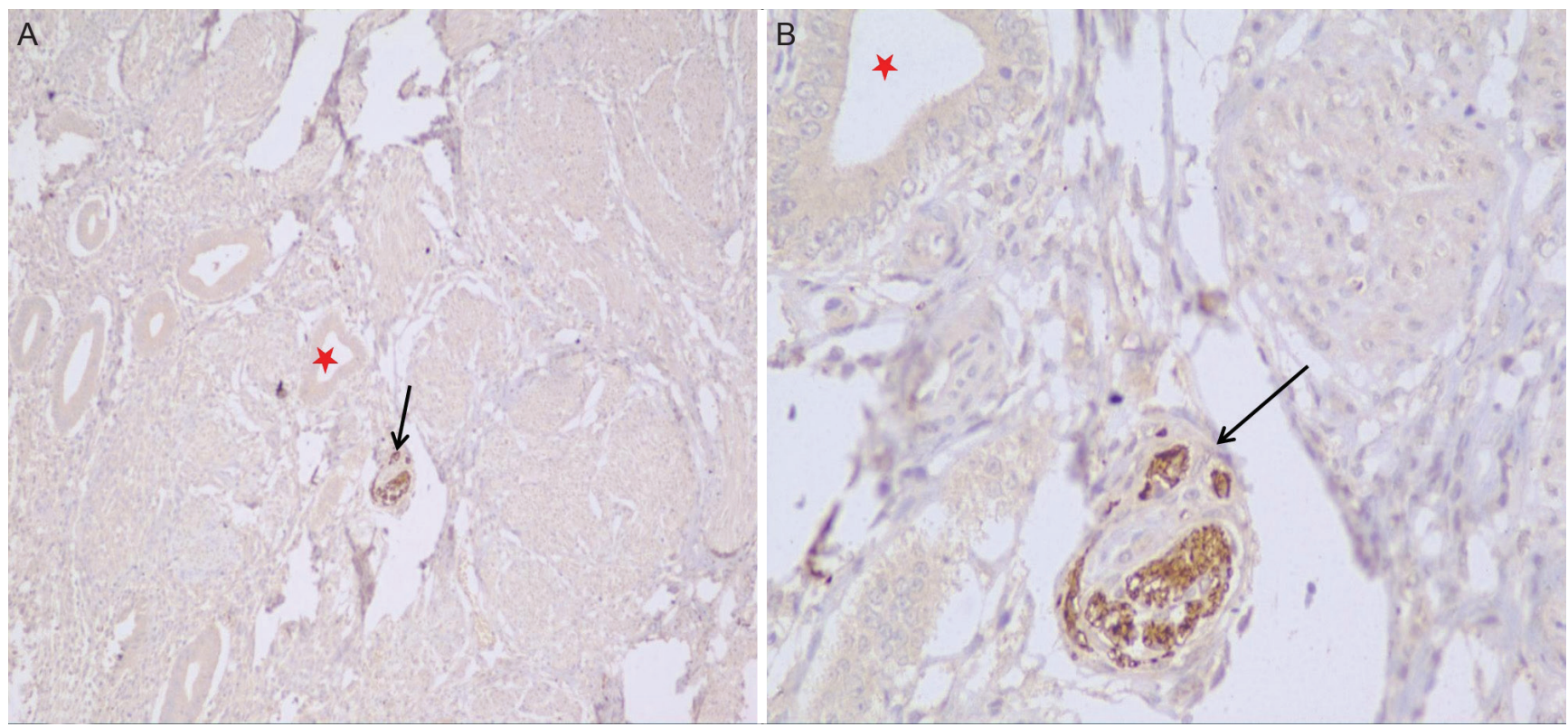

Fig. 4. (A) The endometrium shows nerve fibers highlighted by the protein gene product (PGP) 9.5 antibody (black arrows) in a case of adenomyosis. (B) High power view of the image in (A) where the upper right corner shows the myometrium (red asterisks) (immunohistochemical staining, $\times 40)$. 


\section{Obstetrics \& Gynecology Science}

Garima Yadav, et al. Nerve fibers in eutopic endometrium

any nerve fibers (Table 2). While there was no significant difference in detecting nerve fibers in the endometrium collected through hysterectomy or through endometrial curettage, none of the endometrial aspirates showed any nerve fibers. This can be explained by the fact that in the aspirate tissue, only fragmented endometrial glands from the superficial layer of the endometrium were obtained. Among the 73 women with adenomyosis (including those with coexistent fibroid uterus), 10 (13.7\%) were positive for PGP 9.5, which was again seen in the basal endometrium close to the adjacent myometrium (Fig. 4).

\section{Nerve fiber detection in the endometrium as a diagnostic test}

The sensitivity of nerve fiber detection in the endometrium as a diagnostic test for endometriosis is $55.4 \%$, which increases to $67.39 \%$ if only deep endometrial biopsy is performed; the specificity is $92.7 \%$. The positive predictive value (PPV) of the test is $72.09 \%$ and the negative predictive value (NPV) is $85.87 \%$ (Table 3). The correlation between the VAS score and nerve fiber density in endometriosis was calculated using the Spearman Rank correlation coefficient (rho), which was 0.331 , thus establishing a positive correlation between the nerve fiber density and VAS score (Fig. 5).

\section{The presence of nerve fibers in different phases of the menstrual cycle}

Among 56 women with endometriosis, 37 (66.07\%) were sampled during the proliferative phase and 19 (33.92\%) were sampled during the secretory phase. There was no significant difference in the detection of nerve fibers depending on the type of endometrium in different phases of the menstrual cycle in women with endometriosis $(P=0.471)$.

\section{Discussion}

In the present study, we found that the presence of sensory nerve fibers in the deeper layers of the functional endometrium could correlate with endometriosis with a sensitivity of $55.4 \%$ and a specificity of $92.7 \%$, and the sensitivity of the test improved to approximately $68 \%$ if deep endometrial

Table 3. Comparison of histopathological diagnosis of endometriosis and nerve fiber detection by IHC to assess the diagnostic accuracy of endometrial nerve fibers in the endometrium for predicting endometriosis

\begin{tabular}{lcc}
\hline & Endometriosis confirmed by HPE & Endometriosis absent on HPE \\
\hline Nerve fibers present in endometrium & $31(72.1)$ & $12(27.9)$ \\
Nerve fibers absent in endometrium & $25(14.1)$ & $152(85.9)$ \\
\hline
\end{tabular}

Values are presented as number (\%).

IHC, immunohistochemistry; HPE, hitopathological examination.

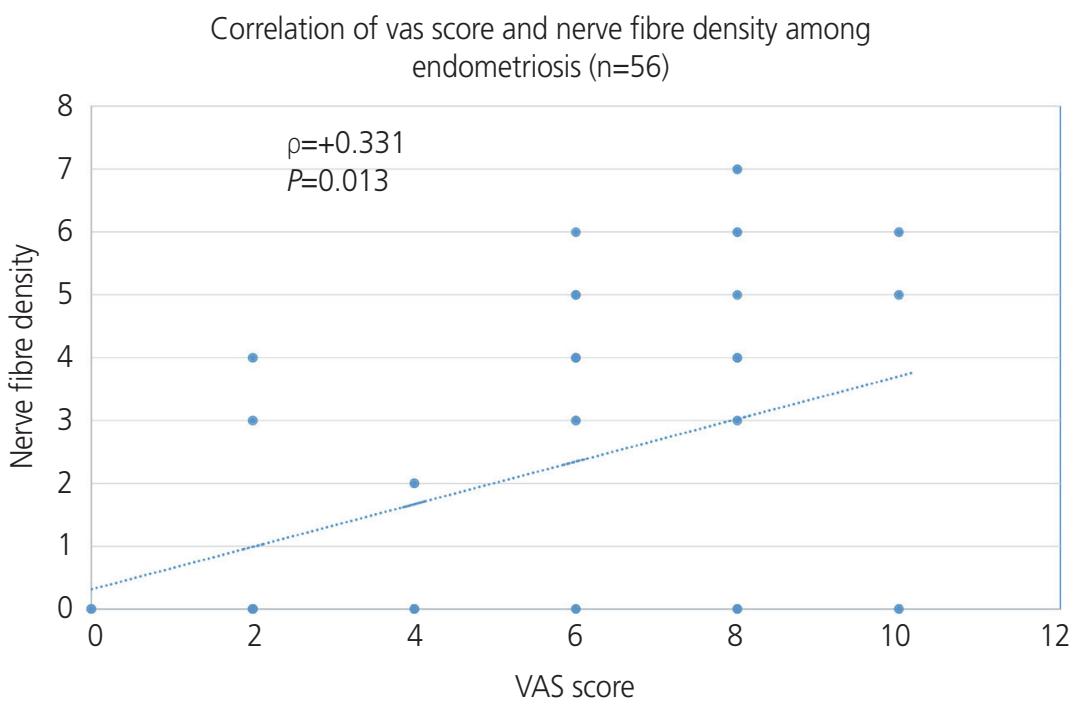

Fig. 5. Correlation of the Visual Analog Scale (VAS) score and nerve fiber density among patients with endometriosis. 


\title{
Obstetrics \& Gynecology Science
}

\author{
Vol. 64, No. 5, 2021
}

biopsy was performed using a uterine curette. These nerve fibers also correlated well with the severity of dysmenorrhea in women with endometriosis, but not with the stage of the disease.

In the last decade, developing a minimally invasive technique to diagnose endometriosis with fair reliability has been the focus of research. In this context, it is highly justifiable to encourage a test that can replace laparoscopy or at least have an acceptable specificity to rule in its diagnosis so that a therapeutic trial is provided to the woman while she awaits surgery or when there is no immediate indication for surgical excision. The most promising results come from the role of nerve fiber detection in the functional layer of the eutopic endometrium of women with endometriosis using various nerve-specific antigens, most specifically the (PGP 9.5), which stains positive in the presence of small nerve fibers using IHC. However, there are heterogeneous results reported by various study groups in terms of the method of collection of endometrium and phase of menstrual cycle, and it is still unclear whether endometrial nerve fibers are pain-specific or disease-specific.

Yadav et al. [6] demonstrated neural elements in the eutopic endometrium of $24 / 30$ cases $(80 \%)$ in women with endometriosis and in none of the controls $(P<0.01)$. They demonstrated a significantly high density of nerve fibers in the endometriomas and peritoneal implants in all 30 cases of confirmed endometriosis. In addition, the presence of nerve fibers in the eutopic and ectopic endometrium was positively correlated with the intensity of pain $(P<0.001)$. Most previous studies have demonstrated similar findings with varying techniques of endometrial tissue collection. Elgafor El Sharkwy [7] also studied nerve fibers in the endometrium of women presenting with infertility and/or pelvic pain with or without endometriosis. They concluded that the presence of nerve fibers in the functional layer of the endometrium detected endometriosis with a high sensitivity and specificity (92\% and $82 \%$, respectively); however, these studies did not include other pathologies, such as uterine fibroids and adenomyosis presenting with dysmenorrhea or pelvic pain.

In contrast to the above findings, few studies $[10,11]$ with varied methodologies in terms of the collection of endometrial tissue, IHC protocol, and comparison groups, show that endometrial nerve fibers are not specific to the diagnosis of endometriosis and neither their presence correlates with the presence or absence of pain symptoms. In their review of the diagnostic use of serum and endometrial biomarkers, Cochrane et al. concluded that PGP 9.5 meets the criteria of a replacement test for endometriosis, but further studies are needed with standardized methodology to confirm its diagnostic potential [12].

In our study, we used the reusable metal uterine curette while taking samples in the operation theatre, and Novak's curette or the Pipelle endosampler, especially in women undergoing endometrial sampling in the out patient department, as it does not require any degree of cervical dilatation. We deliberately chose all the above-described methods because cost effectiveness and the ease of sampling are essential prerequisites for any diagnostic test to become widely accepted in clinical practice, especially in low resource settings. However, we found that the nerve fibers were located in the deeper layer of the functional endometrium and were detected only in the biopsy tissue and not in the endometrial aspirates, irrespective of the phase of the menstrual cycle. We also included a control group of 30 women, who underwent hysterectomy mainly for unexplained abnormal uterine bleeding with or without dysmenorrhea and had no histopathological evidence of any uterine pathology, and found that endometrial fibers were not detected in the endometrium of these women.

In our study, we found that the percentage of women with endometriosis with nerve fibers in their endometrium was significantly higher than that of women with adenomyosis and fibroid uterus or with no local pathology in the uterus. The additional cost of adding IHC (PGP 9.5) in a referral laboratory would be only approximately 100 rupees, which is much less than the cost of diagnostic laparoscopy or MRI. The presence of nerve fibers in the basal endometrium rules in the diagnosis of endometriosis with fairly good specificity; hence, the empirical treatment for endometriosis can be initiated, especially in young symptomatic women with peritoneal disease. The authors recommend that a matched case-control study with multiple biomarkers would be ideal for establishing the diagnostic accuracy of endometrial biomarkers in endometriosis.

\section{Conflict of interest}

No potential conflict of interest relevant to this article was reported. 


\section{Obstetrics \& Gynecology Science}

Garima Yadav, et al. Nerve fibers in eutopic endometrium

\section{Ethical approval}

The study was approved by the Institutional Ethics Committee of All India Institute of Medical Sciences, Jodhpur. The study was performed in accordance with the principles of the Declaration of Helsinki.

\section{Patient consent}

All the participants provided written informed consent to participate in the study.

\section{Funding information}

This study was funded by the Intramural Research Grant of the All India Institute of Medical Sciences, Jodhpur.

\section{Acknowledgments}

We would like to acknowledge Prof. Sanjeev Misra, Director and CEO, AlIMS, Jodhpur for his continuous support and guidance during the project.

\section{References}

1. Ferland LV, Shah DK, Kvaskoff M, Zondervan K, Missmer $S A$. Epidemiological and clinical risk factors for endometriosis. In: D'Hooghe T, editor. Biomarkers for endometriosis. Cham (CH): Springer; 2015. p.95-121.

2. Parasar P, Ozcan P, Terry KL. Endometriosis: epidemiology, diagnosis and clinical management. Curr Obstet Gynecol Rep 2017;6:34-41.

3. Kiesel $L$, Sourouni M. Diagnosis of endometriosis in the 21st century. Climacteric 2019;22:296-302.

4. Nisenblat V, Bossuyt PM, Farquhar C, Johnson N, Hull
ML. Imaging modalities for the non-invasive diagnosis of endometriosis. Cochrane Database Syst Rev 2016;2:CD009591.

5. Kobayashi H, Yamada Y, Morioka S, Niiro E, Shigemitsu A, Ito $F$. Mechanism of pain generation for endometriosis-associated pelvic pain. Arch Gynecol Obstet 2014;289:13-21.

6. Yadav G, Radhakrishnan G, Singh N, Radhika AG. Detection of endometrial nerve fibres-a novel technique to diagnose endometriosis. J Endometr Pelvic Pain Disord 2013;5:144-50.

7. Elgafor El Sharkwy IA. Combination of non-invasive and semi-invasive tests for diagnosis of minimal to mild endometriosis. Arch Gynecol Obstet 2013;288:793-7.

8. Ellett L, Readman E, Newman M, Mcllwaine K, Villegas R, Jagasia $N$, et al. Are endometrial nerve fibres unique to endometriosis? A prospective case-control study of endometrial biopsy as a diagnostic test for endometriosis in women with pelvic pain. Hum Reprod 2015;30:280815.

9. Boujenah J, Hugues JN, Sifer C, Bricou A, Cédrin-Durnerin I, Sonigo $C$, et al. Endometriosis Fertility Index, or classification of the American Society of Reproductive Medicine for postoperative endometriosis patients with infertility: which is more relevant? Gynecol Obstet Fertil 2015;43:806-9.

10. Leslie C, Ma T, McElhinney B, Leake R, Stewart CJ. Is the detection of endometrial nerve fibers useful in the diagnosis of endometriosis? Int J Gynecol Pathol 2013:32:149-55.

11. Bektaş YT, Sakin Ö, Pirimoğlu ZM, Yılmaz AO, Başak K, Büyükbayrak $E E$, et al. Diagnostic value of endometrial nerves in the endometrial tissue of patients with endometriosis. South Clin Ist Euras 2017;28:261-5.

12. Gupta D, Hull ML, Fraser I, Miller L, Bossuyt PM, Johnson $\mathrm{N}$, et al. Endometrial biomarkers for the non-invasive diagnosis of endometriosis. Cochrane Database Syst Rev 2016;4:CD012165. 